\title{
Quantitative analysis of Coordination between Public Transport and Urban Form in Medium or Small Urban
}

\author{
Yang $\mathrm{Li}^{1}$, Yanbo $\mathrm{Li}^{2}$, Wencong $\mathrm{He}^{3}$ \\ 1. School of Highway, Chang'an University, China \\ 2. School of Electronic and Control Engineering, Chang'an University, China \\ 3. Henan Provincial Communications Planning \& Design Institude, China \\ choseoner002@gmail.com, acee2017@hotmail.com, choseone@126.com
}

Keywords: medium or small urban; public transportation; urban form; quantitative analysis of coordination

\begin{abstract}
The public transport and urban form of small and medium-sized cities have entered a rapid development and evolution stage. In order to make small and medium-sized cities to be healthy and sustainable development, this paper makes a quantitative analysis of the coordination of public transport system and urban form in small and medium-sized cities. First, establish the index system which can represent the urban public transportation and urban form. Then the principal component analysis is used to peel off the main factors affecting the development of public transport, taking the comprehensive evaluation value of urban public transport system and urban form as the comprehensive development index of each year. With the help of the evolution relationship of their own development index and the relationship of their interactive evolution trend, the paper carries out a higher level coordinated quantitative analysis of urban public transport facilities, operation and urban form.
\end{abstract}

\section{Introduction}

The public transportation and urban form of small and medium-sized cities have entered a rapid development and evolution stage. In this progress, how to make the public transportation and urban form coordinated development to promote the small and medium cities healthy and sustainable development. It is related to the process of new urbanization and the goal of building a well-off society in an all-round way. In 1994, Frank L. D. and Pivo studied the effects of urban form on urban traffic and found out that urban spatial pattern will lead the development and perfection of traffic system ${ }^{[1]}$. In 2004, Karst T. Geurs and Bert van Wee analyzed the present situation and existing problems of public transportation and urban form from the angle of transportation and land use. In 2012, Lu Guo quantitatively analyzed the coordination between the public transport system and urban form and obtained the coordination degree between the public transport system and the urban form by the selection of indexes and the use of principal component analysis. ${ }^{[2]}$ In 2014, Zhu-Ge C X took urban traffic and land use as the research object, used chaos theory and fractal theory to reveal the complexity of the self-organized evolution and structure of urban traffic and land use system from the angle of time and space in Beijing. ${ }^{[3]}$ 


\section{Evaluation index system}

Before coordination analysis, it is necessary to establish an index system which can characterize urban public transport and urban form. ${ }^{[4,5]}$

2.1 Urban Public Transport System Index System. The urban public traffic system index system is divided into two modules, including hardware facility and operation service.

1) Urban public transport system hardware facility indexes. Number of public vehicles; public traffic network coverage percent; urban center station coverage percent (500 meters).

2) Urban public transport system operation service index. Public traffic sharing rate; public traffic punctuality rate and average transfer coefficient.

2.2 Urban Form Index System. The urban form index system is divided into three modules ${ }^{[6-8]}$, including urban spatial scale, urban economic level and urban traffic development.

1) Urban spatial scale index. The scale of urban land use; the number of per capita construction worker.

2) Urban economic level index. The city population scale; gross domestic product (GDP).

3) Urban traffic development index. The road area per capita; the city road network density; the trunk road average intersection spacing.

2.3 Index System Improvement. Through the further improvement of the index system, the index is independent of each other, and the weight is used to reflect the importance of each index. The most suitable method is principal component analysis.

Suppose there are $n$ samples and $p$ observation indexes $(p<n)$. Then we get the raw data matrix $X=\left(X_{1}, X_{2}, X_{3}, \cdots, X_{P}\right)$, and the correlation coefficient matrix is $R$. The new comprehensive indexes are obtained by linear combination of the original $p$ indexes, and they are $Z_{1}, Z_{2}, Z_{2}, \cdots, Z_{k}$. These new indexes are independent of each other, and the variance is decreasing. Therefore, calculate the eigenvalue of the correlation coefficient matrix is $\lambda_{1} \geq \lambda_{2} \geq \lambda_{2} \geq \cdots \geq \lambda_{P}$. The corresponding unit eigenvector is $l_{1}, l_{2}, l_{2}, \cdots, l_{p}$. Then the $i^{\text {th }}$ principal component is $Z_{i}=l_{i} X \quad(i=1,2,3, \cdots, p)$. Usually choose the top $k$ according to the size of the cumulative contribution. In most cases, the previous principal components can already reflect most information of the original index. The steps are as follows.

Step 1: Original p indexes' standardization.

$$
Z_{i j}=\frac{x_{i j}-\overline{x_{j}}}{\sigma_{j}}
$$

Where, $i=1,2,3, \cdots, n, n$ is the number of sample; $j=1,2,3, \cdots, p, p$ is the number of original index ; $\overline{x_{j}}=\frac{1}{n} \sum_{i=1}^{n} x_{i j}, \overline{x_{j}}$ is average value of the $j^{\text {th }}$ index; $\sigma_{j}=\sqrt{\frac{1}{n-1} \sum_{i=1}^{n}\left(x_{i j}-\overline{x_{j}}\right)^{2}}, \sigma_{j}$ is standard deviation of the $j^{\text {th }}$ index.

Step 2: Calculate correlation coefficient matrix $R$ according to standardized data matrix.

Step 3: Calculate the characteristic root $\lambda$, eigenvector $l$ and contribution rate of the $i^{\text {th }}$ index of correlation coefficient matrix $R . \quad \beta_{i}=\lambda_{i} / \sum_{i=1}^{p} \lambda_{i}$.

Step 4: Determine the principal component $F_{1}, F_{2}, F_{3}, \cdots, F_{k}$ according to the contribution rate.

Step 5: Calculation comprehensive evaluation value. 


$$
F=\left(\lambda_{1} / \sum_{i=1}^{p} \lambda_{i}\right) F_{1}+\left(\lambda_{2} / \sum_{i=1}^{p} \lambda_{i}\right) F_{2}+\left(\lambda_{3} / \sum_{i=1}^{p} \lambda_{i}\right) F_{3}+\cdots+\left(\lambda_{k} / \sum_{i=1}^{p} \lambda_{i}\right) F_{k}
$$

\section{Correlation analysis of evolution}

Since the development process of urbanization tends to $S$ shape development. Similarly, the trend of new urbanization development process also tends to $S$ shape curve development. The development evolution of urban form under the new type urbanization should also follow this trend.

The analysis idea of the coordination of public transport and urban form in medium and small city is as follows.

Firstly, after getting the comprehensive evaluation value of urban form in medium and small city in each year, the scattered plot is fitted with $S$-shaped curve. Then evaluate the rationality of urban form development and evolution through fitting degree and fitting inflection point. And analyze coincidence degree of the fitting trend and curve inflection point on the key time node of the actual development process in medium and small city. Furthermore, verify the rationality and applicability of the quantization method and the quantization results.

Secondly, after getting the comprehensive evaluation value of public transport in medium and small city in each year, the scattered plot is fitted with $S$-shaped curve. Then evaluate the development and evolution of public transportation.

Finally, evaluate the coordination degree of urban form and public transport. And put the scattered plot of the comprehensive development index of urban form and public transport on the same coordinate. Then the scattered plot is fitted with $S$-shaped curve. Analyze the evolution process and coordination relationship of urban form and public transportation according to fitting degree and the relationship of fitting development trend.

\section{Case study}

The basic data are mainly obtain from the China Statistical Information Network, the annual national economic and social development statistics bulletin of Dali, the statistical yearbook of Dali and related supplementary investigation.

\subsection{Acquisition and Processing of Urban Public Transport Indexes in Dali}

1) Further determination of index system and acquisition of data. Combine with the actual situation of Dali, the selection index of public transport in Dali is as follows.

a) Hardware facility. Number of public vehicles $\left(t_{1}\right)$; public traffic network coverage percent $\left(t_{2}\right)$; number of urban center station (500 meters) $\left(t_{3}\right)$.

b) Operation service. Public traffic sharing rate $\left(t_{4}\right)$; public traffic punctuality rate $\left(t_{5}\right)$; average transfer coefficient $\left(t_{6}\right)$.

Index Data are shown in Table I. 
Table I. Evaluation index of public transport system development in Dali

\begin{tabular}{|c|c|c|c|c|c|c|c|c|}
\hline Year & 2008 & 2009 & 2010 & 2011 & 2012 & 2013 & 2014 & 2015 \\
\hline $\mathrm{t}_{1}(\mathrm{veh})$ & 194 & 207 & 234 & 252 & 289 & 328 & 345 & 365 \\
\hline $\mathrm{t}_{2}(\%)$ & 51.4 & 53.5 & 54.2 & 56.6 & 60.2 & 62.9 & 65.5 & 70.2 \\
\hline $\mathrm{t}_{3}$ & 5.3 & 6 & 6.3 & 6.7 & 7.3 & 8 & 8.7 & 9.7 \\
\hline $\mathrm{t}_{4}(\%)$ & 21.3 & 22.5 & 25.6 & 27.8 & 26.6 & 28.2 & 30.4 & 32.7 \\
\hline $\mathrm{t}_{5}(\%)$ & 89.5 & 90.7 & 90.3 & 90.9 & 89.2 & 88.8 & 90.3 & 90.1 \\
\hline $\mathrm{t}_{6}$ & 1.06 & 1.08 & 1.11 & 1.13 & 1.19 & 1.16 & 1.18 & 1.17 \\
\hline
\end{tabular}

2) Further processing of index system. In order to solve the problem of correlation and weight of indexes and achieve the extraction of principal components and obtain the contribution rate of each index, the index need to further process use SPSS software.

a) Standardization of index system. Standardization process the index system according to formula (1). The results are shown in Table II.

Table II. Standardized evaluation index of Public transport in Dali

\begin{tabular}{|c|c|c|c|c|c|c|c|c|}
\hline Year & 2008 & 2009 & 2010 & 2011 & 2012 & 2013 & 2014 & 2015 \\
\hline $\mathrm{t}_{1}$ & -1.28 & -1.08 & -0.66 & -0.38 & 0.19 & 0.79 & 1.05 & 1.36 \\
\hline $\mathrm{t}_{2}$ & -1.21 & -0.89 & -0.78 & -0.41 & 0.14 & 0.55 & 0.94 & 1.66 \\
\hline $\mathrm{t}_{3}$ & -1.32 & -0.85 & -0.64 & -0.37 & 0.03 & 0.51 & 0.98 & 1.66 \\
\hline $\mathrm{t}_{4}$ & -1.47 & -1.16 & -0.34 & 0.24 & -0.08 & 0.35 & 0.93 & 1.53 \\
\hline $\mathrm{t}_{5}$ & -0.64 & 0.98 & 0.44 & 1.25 & -1.05 & -1.59 & 0.44 & 0.17 \\
\hline $\mathrm{t}_{6}$ & -1.56 & -1.14 & -0.52 & -0.10 & 1.14 & 0.52 & 0.94 & 0.73 \\
\hline
\end{tabular}

b) Extraction of principal component. Analyze the principal component of index system use SPSS software. And obtain two principal components, the eigenvalues are 4.716 and 1.032, the variance contribution rates are $78.595 \%$ and $17.199 \%$, and the cumulative contribution rate is 95.794\%. The results are shown in Table III.

Table III. Principal component analysis results

\begin{tabular}{|c|c|c|c|c|c|c|}
\hline \multirow{2}{*}{ Component } & \multicolumn{3}{|c|}{ Initial eigenvalue } & \multicolumn{3}{c|}{ Square load extraction of principal } \\
\cline { 2 - 7 } & Total & $\begin{array}{c}\text { Variance } \\
\text { contribution } \\
\text { rate }(\%)\end{array}$ & $\begin{array}{c}\text { Cumulative } \\
\text { contribution } \\
\text { rate }(\%)\end{array}$ & Total & $\begin{array}{c}\text { Variance } \\
\text { contribution } \\
\text { rate }(\%)\end{array}$ & $\begin{array}{c}\text { Cumulative } \\
\text { contribution } \\
\text { rate }(\%)\end{array}$ \\
\hline 1 & 4.716 & 78.595 & 78.595 & 4.716 & 78.595 & 78.595 \\
\hline 2 & 1.032 & 17.199 & 95.794 & 1.032 & 17.199 & 95.794 \\
\hline 3 & 0.202 & 3.368 & 99.161 & & & \\
\hline 4 & 0.041 & 0.688 & 99.850 & & & \\
\hline 5 & 0.008 & 0.131 & 99.981 & & & \\
\hline 6 & 0.001 & 0.019 & 100.00 & & & \\
\hline
\end{tabular}

Therefore, we can get the principal component matrix which represents the contribution value of each index. It indicates the importance of the index. The principal component load matrix of public transport in Dali is shown in Table IV. 
Table IV. Principal component load matrix

\begin{tabular}{|c|c|c|}
\hline \multirow{2}{*}{} & \multicolumn{2}{|c|}{ Principal component } \\
\cline { 2 - 3 } & 1 & 2 \\
\hline $\mathrm{t}_{1}$ & 0.994 & 0.153 \\
\hline $\mathrm{t}_{2}$ & 0.961 & 0.265 \\
\hline $\mathrm{t}_{3}$ & 0.958 & 0.276 \\
\hline $\mathrm{t}_{4}$ & 0.983 & -0.070 \\
\hline $\mathrm{t}_{5}$ & -0.212 & 0.545 \\
\hline $\mathrm{t}_{6}$ & 0.911 & -0.294 \\
\hline
\end{tabular}

c) Determination of public transport comprehensive development index. By multiplying the principal component load matrix with the standardized index matrix, the score of the principal component in each year can be obtained, as shown in Table V.

Calculate the comprehensive evaluation value of Dali public transport system by using formula (2). The results are shown in Table VI.

The fitting effect of S-shaped curve is shown in Fig. 1. Table VI. The comprehensive evaluation value of Dali public transport in each year

\begin{tabular}{|l|l|l|l|l|l|l|l|l|}
\hline Year & 2008 & 2009 & 2010 & 2011 & 2012 & 2013 & 2014 & 2015 \\
\hline score & -5.169 & -3.978 & -2.307 & -0.906 & 1.064 & 2.215 & 3.686 & 5.393 \\
\hline
\end{tabular}

Table V. The score of principal component of Dali public transport in each year

\begin{tabular}{|c|c|c|c|c|c|c|c|c|}
\hline Year & 2008 & 2009 & 2010 & 2011 & 2012 & 2013 & 2014 & 2015 \\
\hline 1 & -6.430 & -5.130 & -2.920 & -1.246 & 1.535 & 2.957 & 4.563 & 6.670 \\
\hline 2 & -0.668 & 0.315 & -0.068 & 0.425 & -0.827 & -0.637 & 0.579 & 0.877 \\
\hline
\end{tabular}

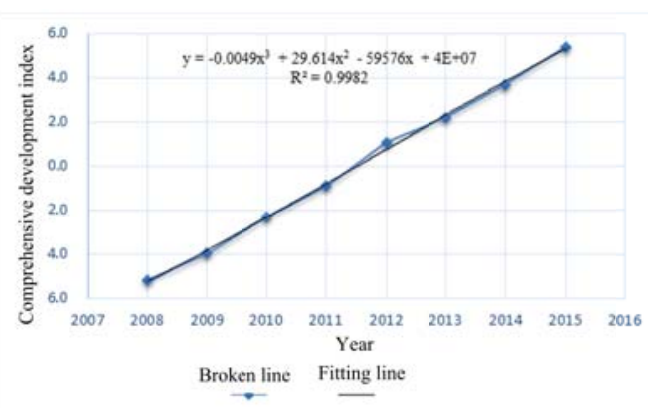

Fig. 1. Evolution trend of comprehensive development index of public transport in Dali

\subsection{Acquisition and Processing of Urban Form Indexes in Dali}

1) Further determination of index system and acquisition of data. Combine with the actual situation of Dali, the selection index of public transport in Dali is as follows.

a) Urban spatial scale index. Urban built-up area $\left(u_{1}\right)$; gross value of construction industry $\left(u_{2}\right)$.

b) Urban economic level index. Urban non-agricultural population $\left(u_{3}\right)$; GDP $\left(u_{4}\right)$.

c) Urban traffic development index. The road area per capita $\left(u_{5}\right)$; the city road network density $\left(u_{6}\right)$; the average intersection spacing $\left(u_{7}\right)$.

Index Data are shown in Table VII.

Table VII. Evaluation index of public form development in Dali

\begin{tabular}{|c|c|c|c|c|c|c|c|c|}
\hline Year & 2008 & 2009 & 2010 & 2011 & 2012 & 2013 & 2014 & 2015 \\
\hline $\mathrm{u}_{1}\left(\mathrm{~km}^{2}\right)$ & 38.18 & 39.3 & 41.3 & 42.9 & 44.5 & 51 & 55.03 & 59.5 \\
\hline $\mathrm{u}_{2}$ (ten thousand Yuan) & 285950 & 323042 & 469624 & 528079 & 689311 & 812426 & 712179 & 840371 \\
\hline $\mathrm{u}_{3}$ (person) & 231412 & 238355 & 241687 & 246865 & 256226 & 305231 & 313368 & 322769 \\
\hline $\mathrm{u}_{4}($ ten thousand Yuan) & 1455033 & 1596433 & 1796838 & 2163220 & 2551705 & 2873274 & 3166506 & 3342100 \\
\hline $\mathrm{u}_{5}\left(\mathrm{~m}^{2}\right)$ & 9.36 & 9.57 & 9.87 & 10.12 & 10.23 & 9.49 & 10.09 & 10.74 \\
\hline $\mathrm{u}_{6}\left(\mathrm{~m} / \mathrm{m}^{2}\right)$ & 5.97 & 6.11 & 6.08 & 6.13 & 6.20 & 5.98 & 6.05 & 6.13 \\
\hline $\mathrm{u}_{7}(\mathrm{~km})$ & 1.16 & 1.11 & 1.08 & 1.05 & 1.03 & 0.91 & 0.87 & 0.85 \\
\hline
\end{tabular}


2) Further processing of index system

a) Standardization of index system. Standardization process the index system according to formula (1). The results are shown in Table VIII.

Table VIII. Standardized evaluation index of urban form in Dali

\begin{tabular}{|c|c|c|c|c|c|c|c|c|}
\hline Year & 2008 & 2009 & 2010 & 2011 & 2012 & 2013 & 2014 & 2015 \\
\hline $\mathrm{u}_{1}$ & -1.06 & -0.92 & -0.66 & -0.46 & -0.25 & 0.58 & 1.10 & 1.67 \\
\hline $\mathrm{u}_{2}$ & -1.39 & -1.22 & -0.53 & -0.26 & 0.50 & 1.08 & 0.61 & 1.21 \\
\hline $\mathrm{u}_{3}$ & -1.01 & -0.83 & -0.74 & -0.60 & -0.35 & 0.95 & 1.17 & 1.42 \\
\hline $\mathrm{u}_{4}$ & -1.26 & -1.07 & -0.79 & -0.28 & 0.25 & 0.70 & 1.10 & 1.34 \\
\hline $\mathrm{u}_{5}$ & -1.26 & -0.80 & -0.14 & 0.41 & 0.65 & -0.97 & 0.34 & 1.77 \\
\hline $\mathrm{u}_{6}$ & -1.41 & 0.37 & -0.02 & 0.62 & 1.51 & -1.29 & -0.40 & 0.62 \\
\hline $\mathrm{u}_{7}$ & 1.31 & 0.88 & 0.62 & 0.37 & 0.19 & -0.84 & -1.18 & -1.36 \\
\hline
\end{tabular}

b) Extraction of principal component. Analyze the principal component of index system use SPSS software. And obtain two principal components, the eigenvalues are 5.271 and 1.460, the variance contribution rates are $75.307 \%$ and $20.852 \%$, and the cumulative contribution rate is $96.160 \%$. The results are shown in Table IX.

The principal component load matrix of urban form in Dali is shown in Table X.

Table IX. Principal component analysis results

\begin{tabular}{|c|c|c|c|c|c|c|}
\hline \multirow{2}{*}{ Component } & \multicolumn{3}{|c|}{ Initial eigenvalue } & \multicolumn{3}{|c|}{ Square load extraction of principal } \\
\cline { 2 - 7 } & Total & $\begin{array}{c}\text { Variance } \\
\text { contribution } \\
\text { rate (\%) }\end{array}$ & $\begin{array}{c}\text { Cumulative } \\
\text { contribution } \\
\text { rate }(\%)\end{array}$ & Total & $\begin{array}{c}\text { Variance } \\
\text { contribution } \\
\text { rate (\%) }\end{array}$ & $\begin{array}{c}\text { Cumulative } \\
\text { contribution } \\
\text { rate (\%) }\end{array}$ \\
\hline 1 & 5.271 & 75.307 & 75.307 & 5.271 & 75.307 & 75.307 \\
\hline 2 & 1.460 & 20.852 & 96.160 & 1.460 & 20.852 & 96.160 \\
\hline 3 & 0.182 & 2.603 & 98.762 & & & \\
\hline 4 & 0.067 & 0.952 & 99.714 & & & \\
\hline 5 & 0.012 & 0.174 & 99.889 & & & \\
\hline 6 & 0.008 & 0.111 & 100.00 & & & \\
\hline 7 & $1.014 \mathrm{e}^{-7}$ & $1.449 \mathrm{e}^{-6}$ & 100.00 & & & \\
\hline
\end{tabular}

c) Determination of urban form comprehensive development index. The score of the principal component in each year can be obtained, as shown in Table XI.

Calculate the comprehensive evaluation value of Dali urban form system by using formula (2). The results are shown in Table XII.

Table XI. The score of principal component of Dali urban form in each year

\begin{tabular}{|c|c|c|c|c|c|c|c|c|}
\hline Year & 2008 & 2009 & 2010 & 2011 & 2012 & 2013 & 2014 & 2015 \\
\hline 1 & -7.048 & -5.263 & -3.350 & -1.482 & 0.725 & 3.050 & 5.180 & 8.189 \\
\hline 2 & -1.396 & 0.449 & 0.359 & 1.174 & 2.003 & -2.419 & -0.921 & 0.752 \\
\hline
\end{tabular}

Table XII. The comprehensive evaluation value of Dali urban form in each year

\begin{tabular}{|l|l|l|l|l|l|l|l|l|}
\hline Year & 2008 & 2009 & 2010 & 2011 & 2012 & 2013 & 2014 & 2015 \\
\hline score & -5.599 & -3.870 & -2.448 & -0.871 & 0.964 & 1.792 & 3.709 & 6.323 \\
\hline
\end{tabular}

The fitting effect of S-shaped curve is shown in Fig. 2.

4.3 Interactive Coordination Evolution Analysis. Through the above fitting trend analysis, it shows the consistency between the development of fitting and the actual process on the critical time node. The fitting effect of S-shaped curve is shown in Fig. 3. 


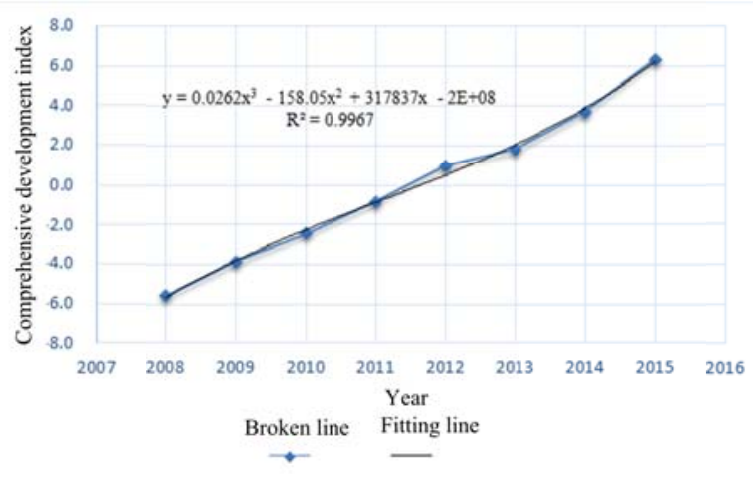

Fig. 2. Evolution trend of comprehensive development index of urban form in Dali

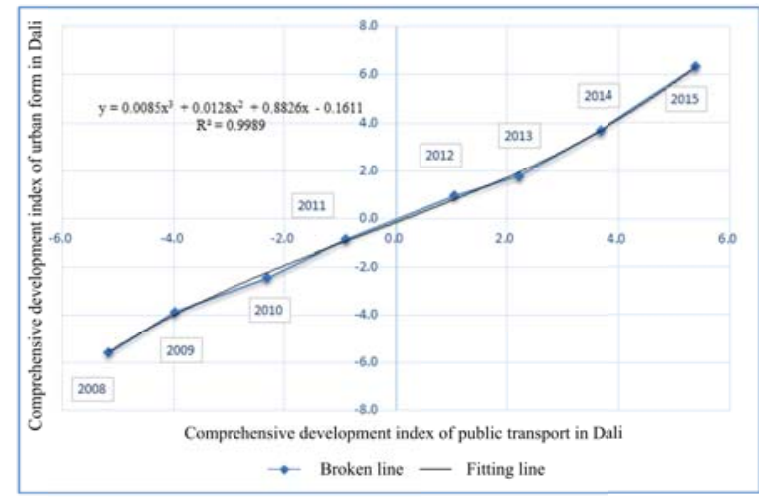

Fig. 3. Evolution trend of interactive development coordination of public transport and urban form in Dali

From the trend map of the evolution correlation between public transport and urban form in Dali, it can be seen that the curve of interactive evolution trend fits well. From the quantitative results, it can be seen that the coordination development shows a change from the public transport leading to the urban form evolution to the urban form evolution leading to the public transport development. The more obvious time node is 2011-2012. Through the above quantitative analysis, it is found that the relationship between public transport and urban form is consistent on the key time node and the trend of development is consistent.

\section{Conclusions}

The research takes the track curve of urbanization development process as the breakthrough point, and in-depth analyzes the main influence factors of the coordination of public transport and urban form under the development of new urbanization. It mainly explains the applicability of the quantitative method and the credibility of the results. The purpose is promoting the coordination development of public transport and urban form. In this study, coordination is stratified for the first time, and different quantitative methods are used for different levels of coordination. This method promotes and supplements the study of the interaction and coordination between public transport and urban form in medium and small city. Finally, analyzed the urban transport of Dali, and indicated that the method is effective. It provides a new idea for the coordination analysis of the development of medium and small city in the future.

\section{Acknowledgment}

This work was supported by Scientific research foundation of Yunnan Provincial Department of Education(2017ZDX006) and Changan University Central University basic Scientific Research Business expenses Innovation team support Project(310832173701).

\section{References}

[1] Frank L D, PIVO G. "Impacts of mixed used and density on the utilization of three modes of travel: the single occupant vehicle, transit, and walking." Transportation Research Board, vol. 1466, pp.44-52, 1994.

[2] Jiang K J, Zhang D Y. "Research on the integrative evolution of urban morphology \& road network based on the fractal theory." Journal of Xinyang Normal University, vol.21, pp.388-391, 2008. 
[3] Zhu-Ge C X, Shao C F, Gao J. "Evolution prospect and structure of urban transportation and land use system." Journal of Transportation Systems Engineering \& Information Technology, vol.14, pp.19-24+32, 2014.

[4] Wang X. "Interaction of urban transit and urban form:implication to urban planning of wuxi." City Planning Review,vol.37, pp.85-88, 2007.

[5] Rui Z S, Fu S C, Hai Y. "Evaluation indices and model of highway transportation sustainable development.” China Journal of Highway \& Transport, vol.18, pp.74-78, 2005.

[6] Guo Y, Qingwen Q I, Jiang L. "Research on the theoretic method and application of the urban form information TUPU.” Journal of Geo-Information Science, vol.13, pp.781-787, 2011.

[7] Narvaez L. "City rules: How regulations affect urban form." Urban Design International, vol.17, pp.351-352, 2012.

[8] Perkins A, Hamnett S, Pullen S. "Transport, housing and urban form: the life cycle energy consumption and emissions of city centre apartments compared with suburban dwellings." Urban Policy \& Research, vol.27, pp.377-396, 2009. 